$\begin{array}{ll}\text { le portiQue } & \text { Le Portique } \\ \text { Revue de philosophie et de sciences humaines }\end{array}$

$10 \mid 2002$

Les paradis artificiels

\title{
Entre ombre et lumière : la rave
}

Jean-Paul Méloni

\section{OpenEdition}

\section{Journals}

Édition électronique

URL : http://journals.openedition.org/leportique/136

DOI : 10.4000/leportique.136

ISSN : $1777-5280$

\section{Éditeur}

Association "Les Amis du Portique"

Édition imprimée

Date de publication : 1 septembre 2002

ISSN : 1283-8594

\section{Référence électronique}

Jean-Paul Méloni, «Entre ombre et lumière : la rave », Le Portique [En ligne], 10 | 2002, mis en ligne le 06 juin 2005, consulté le 08 avril 2021. URL : http://journals.openedition.org/leportique/136 ; DOI : https://doi.org/10.4000/leportique.136

Ce document a été généré automatiquement le 8 avril 2021.

Tous droits réservés 


\title{
Entre ombre et lumière : la rave
}

\author{
Jean-Paul Méloni
}

Introduction

1 Il semblerait qu'à chaque secousse festive, un mouvement de panique s'empare de notre société qui, par crainte d'être envahie par des naïades de divinités s'échappant du monde incandescent des enfers, se rejoue allègrement l'antique scénario de la diabolisation, de l'ensorcellement voire peur archétypale, celui de l'anéantissement des individus qui la composent.

2 L'histoire sociale et culturelle de notre société est traversée par tous ces genres musicaux et festifs tout droit sortis des marges suspectées de contaminer par des liesses collectives ou encore des consommations anarchiques de produits psychotropes toute une partie de la jeunesse.

3 De nombreux travaux qui portent sur le jazz, le rock, le bal populaire comme "défouloir du samedi soir», selon le mot de Jean-Pierre Corbeau, ou encore sur les festivals hippies des années 70, montrent bien la place sensible qu'ont eues ces fêtes, ainsi que les sérieuses condamnations qui vont peser sur elles. La fête techno, à peine installée sur l'autel du divertissement, se voit aussitôt excommuniée et mise d'une certaine façon « sous tutelle ».

4 Du point de vue anthropologique, on a tendance à considérer le corps social comme idéalement garant de l'équilibre entre les forces agonistiques, c'est-à-dire la vie et la mort toujours en frottement dans les moments de réjouissances.

5 Il n'est qu'à voir le récit que fait É. Durkheim lorsqu'il évoque l'annonce d'un corrobori qui désigne un rassemblement festif qui va jusqu'à bouleverser radicalement la vie quotidienne de l'aborigène australien jusqu'à le mettre hors de lui, dans un état de surexcitation. Seule l'issue de la fête, ce moment sensible ou l'effervescence se répand partout et accompagne le groupe qui se sépare pour reprendre ses activités ordinaires parviendra à le calmer, à le ramener à la réalité.

6 Dans ce même sens D. Jeffrey, dans un ouvrage intitulé Jouissance du sacré: religion et postmodernité parle de la fête "comme un moment paroxystique », lors duquel la vie se déplace dans un excès débridé... $»^{1}$. 
7 Devant cet excès la société se voit bien souvent contrainte de réagir et d'anticiper sur un risque toujours probable de débordement, de renversement. Le carnaval comme "fête à l'envers " ${ }^{2}$, qui constitue à cet égard un illustre exemple de fête à risque, signale un autre sens qu'il faut situer de manière concomitante dans la dyade temps de travail et temps de repos.

En effet, cette structure spatio-temporelle qui s'inscrit dans la vie sociale des individus, deviendra plus sensible, plus problématique dès le XIx ${ }^{e}$ siècle, c'est-à-dire au moment où les emblèmes de la cité industrielle et urbaine émergent. Ainsi passé ce temps imparti à la récupération des forces du travail, le temps résiduel consacré à la fête est jugé par la société moderne et rationnelle, comme inutile, une pure perte, « une part maudite ", selon le terme de Bataille (1995), dont l'influence sur toute une catégorie de personnes, en particulier les classes pauvres, sera considérée comme néfaste.

9 En effet, dans ces turbulences urbaines, il est toujours des individus prêts à se laisser divertir par quelques libations au cabaret ou encore par quelques virées dans les bals populaires du Paris nocturne.

10 Par ailleurs, devant le souci très hygiéniste de préserver l'ordre moral et la bonne santé, la domestication des activités festives, ainsi que le contrôle des temps et les espaces consacrées à la fête, demeurent comme une absolue nécessité, pour les pouvoirs publics.

11 C'est ainsi que la réorganisation des fêtes préludes aux formes modernes de divertissement tel que le montre 0 . Ihl ${ }^{3}$, suivant cet impératif s'appuiera pour y parvenir sur la dimension éducative et pédagogique. Accompagner des enfants vers le monde ordonné et sain des adultes et en faire de bons citoyens, voilà qui assigne à la fête une nouvelle fonction.

On observe alors que toute son intervention, ce resserrement du contrôle qu'il soit des corps, des émotions ou des comportements, est indexé à l'usage, pour le moins hiératique, de formules toutes faites qui du simple fait d'être énoncé auraient valeurs cathartiques et comme vertus celles d'éloigner le mal, ou mieux de s'en prémunir.

Rave = Ecstasy : l'équation certes lapidaire a longtemps fait recette, au moins parce qu'elle a nourri différents discours et justifié bon nombre de campagnes de répression.

14 Mais c'est oublier que la Rave est tout à la fois une fête juvénile, une métaphore de la modernité ainsi qu'une production d'une société marchandisée, née des derniers temples industriels et de toute une idéologie qui, de la contrainte au contrôle, a poussé vers la clandestinité toutes formes festives jugées contestataires.

Globalement, la Rave est un acte collectif qui rassemble chaque fin de semaine des milliers de jeunes plongés des heures durant dans des agitations choréiques intenses.

Cette fête de l'actuel, effervescence de l'instant constituent pour de nombreux ravers une expérience profonde et inoubliable.

17 Certains y voient des pures liturgies sacrées, alors que d'autres évoquent une résurgence du monde chamanique, dont le D.J., véritable chamane officiant derrière ses machines, recréerait sur le mode de l'éphémère un nouvel axus-mundi.

18 Ces mondes sensibles, qui sont ici révélés au plus fort de la fête, signalent aussi toute une tension entre un sacré de dissolution ${ }^{4}$, une "sudation du monde" dirait 0 . Cathus ${ }^{5}$ (1998) qui serait le reflet de tout un imaginaire, où se mêlent émotions, sensations, désordre ou encore hallucinations et un sacré de cohésion ${ }^{6}$ apocryphe du 
profane, c'est-à-dire un sacré qui s'est désémantisé, "laïcisé » et rivé au socle du marchand que notre société de consommation a parfaitement su ériger en icône d'un prêt-à-penser.

19 Devenu objet à sacraliser, c'est le marchand, ou au moins le sens qu'on lui prête, qui opère aujourd'hui un savant marquage entre ce qui relève du profane et ce qui tient du sacré jusqu'à les confondre, à les renverser. Les Trend-setters, c'est-à-dire les créateurs de tendance, jouant sur ces confusions, ont acquis un véritable savoir-faire qu'ils développent selon des stratégies commerciales afin d'imposer des modèles culturels, des modes et des styles de vie nouveaux.

Ce partage quelque peu canonique s'impose aux individus selon un scénario devenu aujourd'hui routinier qui se profile derrière tout un processus de récupération de recyclage et d'uniformisation. Il n'est qu'à voir combien ces ready-mades artefacts du corps, de l'art, des idées, pèsent aujourd'hui comme des objets-symboles, des nouveaux objets de cultes (Baudrillard).

21 Le phénomène techno n'a pas échappé à cette standardisation comme en témoigne la théâtralisation de certaines manifestations aujourd'hui labellisés selon cette même procédure et qui apparaît comme un signe d'une culture déjà établie.

Dans ce sens, le raver est donc convoqué à revoir sa conduite, à se conformer. Mais si la Rave est un espace de normalisation, elle sécrète aussi ses propres formes de résistance et de rébellion nourries par tout un esprit de la contre-culture qui plane encore et qui compose l'espace même de l'underground techno.

23 Dans ces lieux de marges, le raver plutôt que de se plier, à cette «culture commercialisée " préfère s'abandonner aux bourdonnements continus d'une musique sans paroles ou alors aux vertiges de danses extatiques, voire plus simplement aux ivresses psychotropiques.

24 Entre une culture qui impose et une culture qui résiste, émerge une fois encore une profonde incompréhension, une tension, entre ceux qui vivent de l'intérieur ce monde des raves et ceux qui de l'extérieur pensent pouvoir maîtriser complètement cette réalité, persuadés que la musique techno est un genre dégénéré, et que la Rave est une entrée dans la toxicomanie.

Dans la première partie, j'aurais l'occasion d'évoquer ce malentendu. Il s'agira de présenter d'autres facettes de la Rave, c'est-à-dire décrire le plus simplement possible la fête devenue ici espace d'initiation, d'expérimentation et lieu de sociabilité.

Pour cela je vais m'appuyer sur une enquête ethnographique, que j'ai réalisés de 1997 à 2000, dans de nombreux événements techno (Raves/free-party, technothèques et technoparades). Ce fut là essentiellement une démarche fondée sur une observation participante périphérique, ou pour le dire à la manière de Colette Petonnet, une observation flottante ${ }^{7}$.

Si cette méthode particulière initiée par les chercheurs de l'école de Chicago offre la possibilité d'une vision rapprochée de la situation, elle comporte néanmoins des obstacles, comme celui de ne pas participer entièrement à certaines activités qui occupent beaucoup de ravers. Je pense notamment à la consommation de produits psychotropes, qui apporte tout un savoir précieux pour la compréhension de la Rave. Pour pallier ce déficit, outre les entretiens que j'ai pu mener moi-même avec plusieurs ravers, je me suis fondé sur différentes études menées dans le cadre de l'O.F.D.T. ${ }^{8}$ ou encore sur une recherche-action produite en 1999 par Médecins du monde '. 


\section{Un peu d'histoire}

aussi la new-wawe ou encore le mythique Krautrock de Kraftwerk, cette musique baptisée techno et House apparaît aux États-Unis dans les premières années de 1980. Très vite elle va connaître un immense succès dans les grands clubs de Chicago, Détroit ou New York. Sécrétée par une culture populaire encore féconde, cette musique se distingue d'autres formes musicales comme le rock et le blues par le fait qu'elle est entièrement conçue à partir de machines électroniques comme le sampler, la boîte à rythme, les platines et l'indispensable table de mixage. Le vinyle, qu'on croyait remplacé par le disc-compact, deviendra la base de la création musicale. De plus ce sont les D.J. (Disc-Jockey), ces anciens pousse-disques, qui se retrouvent aux commandes de ces instruments grâce à un éminent savoir-faire technique, une compétence instrumentale conjuguée à un sens poussé du bricolage artistique ; ces musiciens d'un genre « nouveau » peuvent laisser libre cours à une imagination débordante pour créer des rythmes originaux, authentiques. L'unité de mesure de ces rythmes, c'est le B.P.M, le battement par minute, qui peut, suivant le contexte, être accéléré, comme dans le cas du Hardcore, ou au contraire ralenti comme le cas du style Ambiant.

Cette pratique de création donne lieu à de véritables performances que le D.J. réalise en direct dans un club ou dans une soirée grâce au mix qui désigne dans ce cadre un enchaînement harmonieux ou une superposition adroite de morceaux, afin de créer une onde sonore continue visant à chauffer les corps et à installer dans la fête une ambiance torride. Les clubbers n'auront plus qu'à se laisser divertir, au double sens de s'amuser et se détourner ${ }^{10}$. Cette matière musicale bouillonnante va par la suite se répandre un peu partout en Europe depuis les dancings avant-gardistes d'Ibiza jusqu'aux clubs interlopes de Berlin, réveillant là des clubbers lassés par une disco moribonde.

31 Si aux États-Unis la techno est très vite reconnue comme une musique de revendication, notamment des minorités noires et homosexuelles, en Europe house et techno deviennent des musiques de fêtes notamment en Angleterre ou elles connaîtront des prolongements extraordinaires avec l'accidentel Acid-House. Ce style musical érigé en culture nationale va exercer une véritable fascination sur une jeunesse ravie qui redécouvre le plaisir de la totale. Partant ces liesses irruptives sont aussitôt accusées par les tabloïds anglais de faire l'apologie de l'Ecstasy dont la consommation ne cesse d'augmenter au fur et à mesure que le phénomène des Acid-parties se développe. Ces fêtes, qu'on appelle maintenant des Raves party - nous sommes en 1988 - vont être réprimées par un gouvernement qui les considère comme un danger pour toute la jeunesse. Cette répression affectera par la même occasion tout le mouvement techno qui commence à se structurer autour de tribus nomades, les travellers adeptes de la contre-culture, et noyés dans une idéologie de la contestation inspirée du NewAge, des Hippies et des philosophies orientales. Les travellers feront une large place à la culture de la drogue et de la fête, qu'ils préfèrent organiser eux-mêmes selon le principe du « Do it yourself», le D.I.Y.

32 La France sera très vite absorbée par le phénomène. C'est au début des années 90 que l'on verra apparaître, autour de Paris, les premières Raves-pirates organisées grâce au 
savoir-faire de ces technomades qui, devenus indésirable en Angleterre, se sont exilés dans le restant de l'Europe. Ainsi, grâce à des sounds-systems, ils empruntent la " piste électronique ", selon l'expression de $\mathrm{T}$. Colombie ${ }^{11}$, pour montrer la voie à de très nombreux ravers avec qui ils partagent cette culture de la clandestinité, cette culture du détournement.

Le mouvement techno prend forme et se développe partout en France, attirant de plus en plus de ravers sous le charme de ces ambiances festives et en particulier celles que les ravers rencontrent dans les fêtes sauvages. La prolifération des fêtes clandestines et illégales, leur diabolisation poussent l'état à réagir plus sévèrement. C'est ainsi que des mesures sont adoptées, dans le but à la fois d'accentuer la répression, mais aussi d'assurer une prévention des risques liés à l'usage de drogues.

La Rave-party indexée à la législation en matière de drogue, fait de cette fête un problème de santé publique. Elle sera désormais considérée comme une «soirée Hautrisque » (janvier 1995). Cette volonté de resserrer l'intervention des forces de l'ordre va se poursuivre, et le tout récent vote de l'amendement Mariani visant à interdire les Free-party témoigne bien de cette détermination. Cette prise de position va entraîner des perturbations au sein du mouvement techno. Aujourd'hui, on constate que de nombreux ravers ont regagné les clubs, parce qu'ils ne veulent plus trop prendre de risque. De même pour les petites tribus qui se font plus discrètes dans l'organisation des Free-party. On retrouve en quelque sorte la situation de départ, au sens ou d'un côté, on a le noyau dur de l'underground techno toujours très rebelle et opposé à la société, de l'autre un espèce de mainstream qui s'est considérablement élargi à tout l'espace social. Mais au-delà de cette configuration qui ne cesse d'évoluer, que savonsnous de ces fêtes et pourquoi tant d'hostilité... ?

II. Les Raves : une pratique de détournement

Globalement, le mouvement techno recouvre différentes catégories festives. D'une part la sphère autorisée, visible, du mouvement, qui s'est structurée autour des clubs, des immenses Raves organisées par des sociétés de spectacle, et d'autre part les Parades, formes abouties de la mise en scène techno.

Ces fêtes sont légales et se déroulent dans des lieux appropriés, connus par tout le monde. Ces diverses manifestations forment en soi une culture particulière qui a ses valeurs, ses formes musicales, ses rituels, sa manière d'être que des clubbers ou des Ravers se partagent. Pour certains, ce sont des fêtes qui ont été entièrement récupérées par les majors, par les industries qui en ont fait un loisir marchand.

À l'opposé, une sphère sauvage clandestine a développé tout un concept de fête libre gratuite et cela en toute illégalité. Ce sont les Free-party, les teknivals, certaines Raves, qui se déroulent dans des lieux aussi divers qu'insolites comme un champ, une usine désaffectée, un entrepôt abandonné, une carrière, etc., détournés pour la fête. Le principe est clair, il s'agit pour des tribus, de teufeurs ${ }^{12}$ ou de travellers, de s'approprier l'espace d'une nuit, ou même de plusieurs nuits pour les tekos ${ }^{13}$, d'y installer un décor festif et une fois la fête consommée ou consumée, de quitter les lieux tout aussi clandestinement. C'est là le principe des Zones temporaires d'autonomie développées par un chantre de la contre-culture ${ }^{14}$.

$\mathrm{Vu}$ le caractère illégal de ces manifestations, ces fêtes s'organisent selon un rituel original, qui tient du jeu de piste ou pour certains du parcours initiatique. En effet, ce 
n'est qu'au tout dernier moment et grâce à un système d'info-line que les nombreux ravers avertis par des flyers ${ }^{15}$ pourront rejoindre l'endroit de la fête. C'est là un moyen pour échapper aux gendarmes prêts à intervenir pour interdire la fête. L'enjeu pour les ravers sera bien évidemment d'arriver avant les forces de l'ordre. Lorsque la fête a lieu, le raver peut alors se libérer de ses émotions accumulées durant le jeu de piste. C'est l'occasion pour lui de se lâcher, et de partager avec d'autres de réels instants de plaisir et de passion. Plaisir d'être en fête et se fondre pendant des heures dans des danses profondes et exaltantes qui semblent se perdre dans le temps. C'est là une notion importante pour bien comprendre la problématique des Raves.

En effet, les Raves s'inscrivent dans une temporalité qui est celle imprimée par une société du loisir et de la consommation soucieuse de remplir avant tout le temps libre.

Le temps de la Rave

Les Raves vont se structurer autour de trois moments qui se suivent et qui donnent à la fête cet aspect continu et permanent. Cela rappelle les traditionnelles tournées des bars, la fameuse tournée « des Grands Ducs ».

La Rave donc commence par une Before, c'est-à-dire le temps avant la fête. C'est un temps de préparation, voire d'initiation pour les néophytes, c'est-à-dire ce moment où ils accèdent à un certain savoir, notamment sur les drogues. Ainsi les ravers utilisent ce temps pour se préparer, se mettre en fête, notamment par l'ingestion de stimulants. La Before peut aussi être le moment où les ravers se réunissent dans un bar et en attendant le déclenchement de l'info-line, ils passent le temps à consommer de l'alcool, pour selon certains " chauffer les corps ». L'arrivée dans la rave est le signe de la fête elle-même, moment sensible, excitant car attendu et imaginé, avec ce que cela suppose comme frustration si la fête est annulée. La Rave proprement dite est le corps de la fête. Plus long, plus intense, c'est l'occasion pour le raver de se perdre dans les vertiges d'une ambiance survoltée, électrique. Pour le raver pas question de perdre du temps. Pris dans la tourmente festive, il redoute par-dessus tout le décalage, le contretemps voire l'arrêt du temps, à l'exemple du Cut ${ }^{16}$ ou l'arrivée des forces de l'ordre comme cela se produit parfois dans les Free-party.

43 Le corps est parfois un obstacle à cette immersion dans la fête. Pris là dans la métaphore de la machine, le corps doit être chaud pour pouvoir s'élancer dans la frénésie. La première partie du set musical s'appelle d'ailleurs un Warm-up, réalisé par un D.J. en général débutant ou encore le D.J. résident, qui a pour fonction de réchauffer la salle. Le raver, dans son souci de consommer entièrement la fête, se met à danser, et très vite il est pris dans le mouvement, son rythme s'accélère, il devient plus rapide avec des gestes saccadés, robotisés, rendus plus vifs par une musique tonitruante, une lumière stroboscopique. C'est là, au cœur de la vague, qu'il se laisse « emporter par le mix », comme le rappelle si bien M. Jouvenet ${ }^{17}$.

L'after est le dernier moment de la rave, qui se déroule soit sur place, soit dans un autre lieu comme un café, un club spécialisé dans ce type de fête. C'est généralement un temps plus calme ou le raver se remet d'une intense agitation. C'est là qu'il prend le temps de se retrouver, de communiquer. Les corps se reposent, se détendent. Parfois il dort. Lorsqu'il est en descente de drogues, le raver se met alors en condition pour contrôler des effets qui peuvent être mal ressentis.

Pour d'autres, c'est au contraire l'occasion de repartir dans la fête. Ils prennent alors un stimulant et tentent de retrouver cette frénésie qui les a secoués. La rave est un lieu de profonde exaltation. De nombreux ravers parlent même de transe, notion sensible et 
complexe qui touche au sens originel du mot rave qui signifie «délirer, battre la campagne, divaguer, s'extasier, être déchaîné, être emporté, être ravi... ». La transe nous renvoie au champ des États modifiés de la conscience (E.M.C.), que les ethnologues et d'autres sociologues du rite ont parfaitement bien décrit. G. Lapassade parle des E.M.C. comme d'une expérience au cours de laquelle «...le sujet à l'impression que le fonctionnement habituel de sa conscience se dérègle et qu'il vit un autre rapport au monde, à lui-même, à son corps, à son identité... " ${ }^{18}$.

Parler de la fête comme d'une expérience de modification de la conscience, c'est redire à la suite de R. Bastide (2000), qu'en réalité c'est la fête dans sa totalité qui détermine l'entrée en transe.

Cette « conduite du corps » qui accompagne le raver dans ce dérèglement des capacités mentales, cette dissociation peut-être déclenchés par plusieurs facteurs. Tout d'abord la musique qui intervient directement comme un inducteur hypnotique.

Ainsi le rôle éminent complexe du D.J., la particularité de la matière, la contexture, que $\mathrm{N}$. Abraham définit comme "l'ensemble des circonstances, situations, atmosphère, contexte..., qui entre en synthèse avec le médium pour en déterminer la signification... ${ }^{19}$, favorise l'entrée dans la transe.

C'est là une technique " archaïque... » parfaitement bien maitrisée par les chamans qui, grâce au battement régulier du tambour, deviennent selon les termes de G. Rouget: «les musiquants de leur propre transe... ${ }^{20}$.

Les drogues :la part maudite de la Rave

50 Mais l'observation de ces figures du ravissement qui saisissent ces corps nous amènent à évoquer l'induction psychédélique qui renvoie à un certain usage de psychotropes.

51 Dans le contexte de la Rave, nous le disions c'est une dimension importante. Au-delà de cette vision souvent teintée de catastrophisme qui agite de l'extérieur le corps social, on repère aisément - et du reste le discours des ravers nous y invite -, la place singulière que prennent les drogues dans les pratiques du raver.

52 Une première observation est à faire quant aux lieux ou se déroule la fête. Là, les produits proposés ne sont pas les mêmes suivant que l'on parle de club ou de Freeparty.

53 Dans les clubs qui sont des lieux fixes, fortement contrôlés et où l'alcool est un psychotrope légal très valorisé, ce sont surtout des produits comme la cocaïne et l'ecstasy qui sont proposés, car d'un usage facile. Pour autant la consommation doit se faire discrète, à l'abri du personnel, sous peine de fermeture de l'établissement. Les transactions s'effectuent le plus souvent à l'extérieur, ou parfois dans les toilettes.

Cette situation semble aujourd'hui se modifier eu égard au contexte de répression qui amène, dans les clubs, une clientèle issue du monde de la Free-party. On constate d'ailleurs, au-delà de cette mixité, une hydration des pratiques au sens ou les ravers vont être très vite associés aux rituels qui valorisent l'alcool à forte dose. Ces jeux de compétition entre ceux qui s'alcooliseront le plus rapidement, n'est pas sans conséquence sur ces jeunes, car dès qu'ils se rendent en Free-party, certains vont transporter avec eux ces pratiques qu'ils réaliseront non plus seulement avec de l'alcool, mais avec une quantité de drogues très accessibles.

En Free-party, lieu de toutes les mobilités, de toutes les errances, le marché est plus ouvert, avec la possibilité de trouver une gamme très variée de produits. 
56 Dans ces lieux, la recherche de produit devient, pour le raver-consommateur, une activité importante. Il s'agit avant tout de trouver un bon produit, avec cela s'entend un effet garanti. Dans ces aires culturelles, certaines zones se transforment alors en point de vente, où s'affairent de nombreux revendeurs, venus là pour écouler leurs marchandises. Il y a deux sortes de vendeurs. Tout d'abord ceux que l'on appelle la Racaille ou la Caillera, et qui sont considérés comme des détracteurs de la fête. Ce sont généralement des jeunes venus de banlieues qui écoulent toutes sortes de produits, pas toujours de bonne qualité. Ces vendeurs qui ne sont pas engagés dans la fête fonctionnent à partir de coups de vices et d'arnaques, notamment à l'égard de jeunes teufeurs, pas encore familiarisés avec ce genre de transaction.

On trouve aussi d'autres vendeurs qui sont en fait des teufeurs et qui vendent des produits pour obtenir quelques bénéfices pour rembourser les frais engagés dans la fête.

Le marché des drogues s'organise selon des règles relativement bien définies, que chaque interactant essaie de respecter le mieux possible et cela surtout s'ils sont expérimentés. Un revendeur peut reconnaître "un touriste», catégorie de raver apparenté à un curieux au sens négatif du terme, à qui il proposera un produit de seconde qualité, réservant le bon produit pour le vrai teufeur pour lui-même ou encore pour l'un de ses assistants. De la même façon il sait très vite s'il a affaire à un teufeur ou à un inspecteur de police en civil.

59 Ainsi la consommation pourra se faire selon la disponibilité du produit, sa qualité, le prix de vente. La rave est souvent un terrain d'imitation pour le jeune raver qui peut ainsi faire l'apprentissage des produits et des rituels associés. C'est le plus souvent en groupe que s'effectue cet apprentissage mais parfois c'est un raver plus expérimenté qui va lui faire partager son savoir.

60 À ce niveau, on peut aisément considérer le raver comme un véritable expert des drogues. Il connaît les produits, leurs effets. Son corps dans la plupart des cas lui sert de laboratoire au sens ou il expérimente divers produits sans toujours savoir ce qu'il prend.

61 Ainsi M. qui a consommé de l'ecstasy pour la première fois à l'âge de 16 ans, alors qu'il se rendait dans une fête techno, raconte : « moi ça fait presque quatre ans que je vais en teuf. Encore aujourd'hui je prends mon pied. Bien sûr, à chaque fois que je sors dans une fête, je consomme, surtout des ecstasy, je préfère car j'aime surtout danser. J'ai déjà goûté un peu de tout : du Speed, des Hoffmann, un peu d'héro, en sniff et puis du C. de la coke quoi. Mais tu sais, je prends pas n'importe quoi, je fais gaffe chez qui je le prends. Bon, c'est vrai, tu peux pas toujours savoir ce qu'il y a dedans, mais tu sais, t'es très vite au courant de ce qui faut prendre ou pas... Au début j'essayais de tout, je voulais voir ce que ça faisait. Là maintenant ça y est, j'assure juste pour la fête... » ${ }^{21}$.

62 Dans le contexte festif, le raver évoque plusieurs raisons à sa consommation. Tout d'abord, s'agissant d'une activité qui demande un fort investissement physique, le raver choisira plutôt des produits stimulants, qui apportent de l'énergie.

Afin d'accroître ses capacités et ses performances, notamment en danse, il «mangera » un ecstasy ou sniffera de la cocaïne, du speed. En revanche, s'il veut se construire un délire, un rêve ou encore laisser courir son imagination, il consommera plutôt des produits qui « rendent Love » c'est-à-dire des produits hallucinogènes comme le L.S.D., la Ketamine ou encore toutes sortes de champignons hallucinogènes. Enfin, s'il se sent 
fatigué, souhaite se détendre ou encore accompagner la descente de certains produits, il prendra des relaxants, des opiacés, des drogues de «coma " comme l'héroïne, le rachacha.

Les drogues sont ainsi consommées selon leurs effets ou selon des effets que le raver souhaite ressentir. On observe par ailleurs, dans ce contexte festif, des pratiques de poly-usage. De telles conduites demandent souvent une certaine connaissance et une grande expérience car on ne mélange pas n'importe quel produit à n'importe quel moment de la soirée. Si le mélange d'alcool, tabac, cannabis est le plus courant, d'autres mixtures sont consommées par des ravers devenus experts dans l'art de cuisiner les «poisons ». Le plus connu c'est le speedball, l'équivalent du « cercueil » au niveau de l'alcool qui est un mélange d'héroïne et de cocaïne, pratiqué il y a encore peu par les adeptes du Rock, ou du Heavymétal. Le "Calvin Klein », coktail composé d'un mélange de cocaïne, de kétamine et de speed, est de plus en plus prisé en milieu festif.

Les pratiques de poly-usage reposent sur deux principes. D'une part ce mélange comprend plusieurs produits d'un même genre, aux effets agonistes, deux relaxants par exemple. D'autre part, cela peut être l'association de plusieurs produits d'un genre opposé, c'est-à-dire des produits aux effets antagonistes, comme le mélange d'un stimulant et d'un relaxant. Le poly-usage prend aussi une signification particulière selon les motivations exprimées par les ravers.

C'est souvent alors pour augmenter l'action du produit, ou encore mieux ressentir le trouble lorsque le psychotrope agit dans l'organisme. De plus, ce qui est visé, c'est la rapidité et l'intensité avec lesquelles le produit va se répandre dans le corps. Cette dimension, qui touche au registre du physiologique, c'est-à-dire à la perturbation de l'activité mentale, nous permet de préciser que d'une certaine manière la transe du raver s'inscrit aussi dans une temporalité ainsi créée par le fait même que la conscience soit modifiée.

Dans ce sens l'action du produit s'articule autour de trois moments.

Une fois le psychotrope ingéré, s'opère peu à peu ce que l'on nomme la montée, c'est-àdire ce moment ou la drogue se répand dans le corps, pour atteindre le cerveau et livrer un à un ses multiples effets. Le raver se laisse alors porter, voire plutôt transporter dans un ailleurs, une autre dimension, «ça c'est géant, me disait cette jeune raveuse, surtout quand tu es pris dans la musique, le mouvement. Tu ne vois plus rien autour de toi, t'as l'impression d'être légère et que tu décolles; tu traverses des champs, des forêts, tu survoles la salle... t'es ailleurs quoi... » ${ }^{22}$.

Le raver semble vivre comme s'il était dans un cocon, bercé par ce rythme, qui même accéléré, saccadé, l'installe dans une douce atmosphère. C'est la phase de plateau qui peut durer entre une et deux heures suivant la nature du produit, puis peu à peu les effets se dissipent, le raver reprend conscience de son corps, il revient à la réalité, c'est la descente.

70 Certains ravers pris par l'ambiance peuvent vivre plusieurs fois ces séquences.

71 Ces diverses phases qui troublent le raver, à chaque consommation de psychotropes, plongent ou redoublent une autre modification de la conscience, cette fois provoquée par la matière musicale. À ce niveau, les dimensions musicales et psychotropiques sont intrinsèquement liées.

72 D'une certaine façon, l'introduction hypnotique va suivre un même itinéraire temporel, orchestrée par un D.J. habile qui peut amener les danseurs à vivre ces mêmes 
expériences ${ }^{23}$. À ce niveau, le D.J. construit son set en trois temps. Tout d'abord une phase de montée, c'est-à-dire ce moment ou les corps se mettent au diapason de la musique. Peu à peu le rythme s'accélère, les B.PM. deviennent plus puissants. Le corps pris dans le mouvement se laisse entraîner dans une cadence martelante et qui se répète inlassablement jusqu'à se fondre dans un vertige. Vers la fin de ce cycle, le DJ commence à ralentir progressivement le rythme, les B.P.M. deviennent moins agressifs, cela permet au raver de redescendre doucement, de se remettre de ses émotions.

Les multiples fonctions des drogues

Ces moments d'accélération laissent chez certains ravers une impression, une sensation agréable qu'ils veulent renouveler le plus souvent possible. Pour eux, ces ivresses musicales et psychotropiques relèvent d'une expérience marquante, pour autant que leur corps voudra ou pourra bien tenir le rythme.

Aussi certaines drogues seront consommées à des fins de dopage, notamment les stimulants en raison de leur charge dopaminergique, comme l'ecstasy, la cocaïne, les amphétamines. À ce niveau, des comportements spécifiques semblent émerger dans la recherche de sensations. En effet certains ravers tentent d'atteindre ces "paradis artificiels» par une consommation de produits qu'ils nomment familièrement des « drogues sympas » comme l'ecstasy, le haschich, le speed.

De plus, ces drogues assurent une fonction de relâchement des corps et dés-inhibitions, ce qui favorise les contacts entre ravers.

L'effet entactogène que l'on retrouve dans ces designers drugs vise au fond à se rapprocher, à créer du lien, un être-ensemble où chacun éprouverait du plaisir à partager avec d'autres certaines émotions et participer tous ensemble à la recherche de valeurs hédonistes comme la paix, la tolérance ou la liberté. Cela se retrouve déjà au niveau de la consommation des drogues qui donne lieu à un partage très ritualisé des drogues à consommer. C'est là un principe bien connu, où chacun va offrir une part de produit aux autres membres du groupe (le joint qui circule, par exemple), qui à leur tour rendront la politesse. Dans un contexte de convivialité les ravers tirent des lignes, comme d'autres tirent des pistes, en référence aux rituels des tournées d'alcool chez les jeunes bretons ${ }^{24}$.

77 En revanche, d'autres ravers parmi les plus jeunes cherchent littéralement à s'éclater le corps, à se déchirer le cerveau. La motivation est au contraire animée par l'envie de se défoncer et laisser le corps se diffracter dans la tourmente festive. Ces comportements à risque, que l'on retrouve surtout chez les adolescents, se manifestent à travers des rituels ordaliques.

Au-delà de son lien intrinsèque avec la dimension de la transgression, l'ordalie renvoi au dépassement de soi, à la confrontation avec la mort, au franchissement de ses propres limites, ainsi qu'à l'immortalité. D. Lebreton, dans son ouvrage sur la passion du risque, écrit "...qu'il s'agit dans tous les cas d'affronter la mort sur un mode métaphorique, d'en rendre la menace virtuellement accessible et de l'esquiver en prenant les précautions d'usage. Fixer ainsi la mort, y tracer les limites de sa puissance, renforce le sentiment d'identité de celui qui ose le défi... » ${ }^{25}$.

L'acte même de gober un comprimé d'Ecstasy, dont on ne sait s'il contient du M.D.M.A ou si c'est une molécule chimique aux effets nocifs pouvant même entraîner la mort, n'est pas sans agiter les mêmes troubles de ce rituel ordalique. 
80 Au-delà des effets du produit, les ravers s'expriment assez aisément sur la perception qu'ils ont du produit. Si la Cocaïne est considérée comme un produit de seigneur, de riche, le Speed est la poudre du pauvre. Suivant cette idée, le Cannabis et l'Ecstasy sont souvent perçus comme des produits propres, des bons produits : les «safe drugs ", alors que la Kétamine, en raison des effets très distordus qu'elle produit sur le corps, ou encore l'héroïne, sont parfois très mal vus. De même, en ce qui concerne la façon de les consommer ; l'injection sera rejetée comme une pratique négative, qui ne correspond plus à l'esprit de la fête. C'est d'ailleurs pour cela que cet usage peu visible dans l'espace même de la fête se déroule soit dans la voiture, soit clandestinement à la marge de l'espace festif.

81 Enfin, les ravers évoquent aussi certains produits considérés comme très rares dans le milieu festif. Comme souvent dans les micro-lieux, diverses informations circulent concernant soit un D.J. talentueux, soit un type de fête qu'il ne faut pas manquer, soit encore une drogue qui vient de faire son apparition, et dont les effets seraient surprenants. Ainsi, comme c'est le cas pour le "Beaujolais nouveau», de nombreux ravers attendent impatients que le produit soit disponible pour pouvoir y goûter. Dans ce sens, la curiosité, l'envie de toucher à toutes sortes de produits est une motivation qui revient comme une pratique positive. Les exemples du GHB ${ }^{26}$, du P.C.P. ${ }^{27}$, un produit proche de la mescaline ou encore le D.M.T. ${ }^{28}$ sont à cet égard tout à fait significatifs.

82 Même s'il dit avoir conscience des risques qu'il prend, le raver n'arrive pas toujours à gérer sa consommation. Certains passent d'ailleurs très vite d'une consommation dite récréative à un usage plus régulier, plus nocif. La plupart décident d'arrêter cette consommation, estimant qu'ils en ont fait le tour et qu'ils souhaitent maintenant envisager la fête sous un autre angle. D'autres font carrière dans le produit, c'est-à-dire qu'ils en viennent à consommer dans d'autres contextes, comme sur le lieu de travail, à la fac, ou encore plus simplement chez eux. Certains décident parfois de se professionnaliser en devenant eux-mêmes vendeurs de produits. Dans un but économique, ils souhaitent bénéficier des revenus complémentaires aux aides qu'ils ont déjà. Dans le contexte festif, certaines consommations peuvent aussi entraîner de réels problèmes de santé ; ce sont des malaises, des pertes de conscience ou encore des «bad trips ", qui désignent un malaise psychologique sous l'influence d'un ou plusieurs produits. Ils peuvent alors solliciter des unités médicales de plus en plus présentes, qui les prendront en charge, dans des chill-out sanitaires spécialement aménagés à cet effet.

La Rave est à ce propos un lieu très investi par différents acteurs dont l'intervention vise à contrôler ces lieux à risque, et à y inscrire une activité de prévention. Ce sont des associations comme Médecins du monde, la Croix Rouge, ou alors des acteurs de la prévention des toxicomanies qu'ils soient issus du champ professionnel ou du monde des ravers eux-mêmes à travers des associations de santé communautaires (Technoplus, Spiritek, le tipi, etc.).

84 Les discours produit par ces différents intervenants ne peuvent rendre compte que partiellement de la réalité des raves, ce qui parfois crée des tensions et des malentendus avec les ravers qui réagissent dès lors qu'ils se voient stigmatisés et taxés de toxicomanes.

85 Pour autant, ce qui surprend dans cette logique, c'est le fait que sur le nombre de ravers qui consomment des produits en rave, très peu seulement viennent consulter 
dans les Centres Spécialisés. C'est là d'ailleurs un grand problème pour tous les intervenants.

Au-delà de ces quelques considérations livrées ici au pas de charge, je voudrais en guise de conclusion ouvrir sur quelques pistes de réflexions.

La Rave, nous l'avons dit, est un miroir de notre société, un reflet de la modernité. À ce niveau elle ne fait que révéler des pratiques, qui sont sinon valorisées du moins encouragées, qu'il s'agisse de drogues à la carte, de Designers Drugs, de Danse-Pills, de produits de dopage. Ces drogues de la performance, ces prothèses chimiques sont dans notre société promises à un bel avenir, ne serait-ce que, comme le dit A. Ehrenberg (1991), pour éviter l'effondrement psychique et physique de l'individu.

Si les drogues de synthèse ouvrent encore sur d'autres dimensions de notre monde actuel, comme la vitesse ou la cyberculture, elles trouvent un écho dans le vaste projet des Technosciences.

Pris dans cette perspective, la rave pourrait être considérée comme un objet hybride et le raver comme une chimère, avec un corps de sauvage harnaché du masque de la technique. C'est là un transfuge de ce corps, devenu un technocorps, et par l'artefact de la modernité.

90 L'Adieu au corps (D. Lebreton, 2000), signale ici une nouvelle dérive, une mutation qui obligerait chacun à s'adapter, voire "à s'inventer" à nouveau. Et si dans cette turbulence festive, le regard du raver n'était autre que l'expression de son abandon, le rictus de sa propre mort... ${ }^{29}$

\section{NOTES}

1.. D. JEFFREY, Jouissance du sacré : religion et postmodernité, 1998, p. 127.

2.. Daniel fabre, Carnaval ou la fête à l'envers, Paris, Découvertes Gallimard, 1992.

3.. Olivier IHL, La Fête républicaine, Paris, Gallimard, 1996.

4.. Sacré de dissolution et de cohésion sont des notions bien mises en évidence par Roger CAILloIs dans son ouvrage : L'Homme et le Sacré, Paris, Gallimard, Folio, 1950. Si le sacré de dissolution touche à la transgression, au désordre, au vertige donc à la fête, le sacré de cohésion, contient en lui-même le sens de stabilité, de profane et d'harmonie. 5.. Olivier CATHus, L’Âme sueur, le Funk et les Musiques populaires du $X^{e}$ siècle, Paris, Desclée de Brouwer, 1998.

6.. Voir note 4.

7.. Colette PETONNET, On est tous dans le brouillard, Paris, Galilée, 1979.

8. Les études réalisées sous l'égide de l'O.F.D.T. sont nombreuses. On peut signaler, celles conduites par T. COMBINÉ, Nacer LALAM, et M. SCHIRAY, « Les filières produites psychotropes à partir des sacrées de musique techno ", octobre 1999 (étude $n^{\circ} 15$ ), ou encore le remarquable travail réalisé par un groupe de chercheurs "Pratiques et représentations émergentes dans les champs de l'usage de drogues en France », OFDT, 2001. 
9.. Je fais référence ici au rapport de recherche action « usager de drogue de synthèse (Ecstasy, L.S.D., danse-pills, amphétamines...) » réalisé par Médecins du Monde, Mission Rave D.G.S., octobre 1999.

10.. Voir Y. C. ZARKA, 2001, n 7, p. 3.

11.. Thierry colombie, Technomades, la piste électronique, Paris, Stock, 2001.

12.. Ce sont ceux qui participent à la culture free partie, ou de l'underground. La culture techno a produit diverses entités socioculturelles comme le Clubber attaché au monde des clubs, les ravers, etc.

13.. Tekos : terme familier pour dire technival : ce sont des fêtes interdites qui durent plusieurs jours. Cette catégorie festive est intéressante. La saison des technivals débutent en général au $1^{\mathrm{er}}$ mai pour se terminer vers fin septembre.

14.. T.A.Z. : zones temporaires d'autonomie que l'auteur définit comme « ...une insurrection sans engagement direct contre l'état, une opération de guérilla qui libère une zone (de terrain, de temps, d'imagination) puis se dissout avant que l'état ne l'écrase pour se reformer ailleurs dans le temps ou l'espace. Puisque l'état est davantage concerné par la simulation que par la substance, la T.A.Z. peut « occuper » ces zones clandestinement et poursuivre en paix relative ses objectifs festifs pendant quelques temps... » (H. BEY, T.A.Z., 2000, p. 13- 14). H. Bey est aussi connu pour être un philosophe de la contre-culture.

15.. Flyers : carton d'invitation, qui annonce un événement festif.

16.. Le cut : c'est la coupure de courant, l'arrêt de la musique qui provoque souvent une déception chez les ravers. Le cut peut se produire suite à une intervention de la police. 17.. Morgan JOUVENET, «Emportés par le mix », Revue Terrains n 37, Paris, Éd. du Patrimoine, 2001, p. 45.

18.. G. LAPASSADE, 1987, p. 5.

19.. Nicolas ABRAHAM, Rythmes, Paris, Flammarion, 1999, p. 85-41.

20.. Gilbert ROUGET, La Musique et la Transe, Paris, Gallimard, 1990.

21.. M., Entretien, Mai 1999.

22.. L., Entretien, octobre 1998.

23.. J'ai évoqué cette problématique lors d'une intervention portant sur la transe, à l'occasion d'une rencontre organisée par l'ERASE en novembre 1999.

24.. Voir à ce sujet J. GENDREAU, 1998.

25.. D. LEBRETON, 1991, p. 17.

26.. G.H.B. Gama- OH ou Ecstasy liquide Anesthésiant conçu par Labori en 1990 et utilisé dans le traitement de la narcolepsie et de l'alcoolisme.

27.. Anesthésique proche de la Kétamine, encore appelé Angel Dust P.C.P., produit conçu en 1950 pour le traitement de la narcolepsie et de l'alcoolisme.

28.. D.M.T : produit hallucinogène que l'on retrouve dans certaines plantes ou dans les sécrétions de certains crapauds. Synthétisé en 1931, il est surtout utilisé à partir des années 50 dans nos sociétés (il est connu et utilisé par les Amérindiens depuis plusieurs siècles...).

29.. Bibliographie complémentaire :

AN-Ju, Techno, ed. Hors Collection, Luçon 1998 ; Guillaume BARA, La Techno, Librio musique, Paris 1999 ; Philippe BIRGY, Mouvement techno et transit culturel, Paris, L'Harmattan, 2001 ; Ecstasy, Des données biologiques et chimiques au contexte d'usage, INSERN 1997 ; Astrid FoNTAINE, Caroline FonTANA, Raver, Paris, Anthropos, coll. « Poche », 1996 ; Michel GAILLOT, La Techno. Un laboratoire artistique et politique du présent, Paris, Dis 
voir, 1999 ; Joseph GHOSN, « Du « Raver » au « Sampler » : vers une sociologie de la techno », in Revue L'Homme et la Société, L'Harmattan, 1997/4, p. 87-98 ; Anne-Marie GREEN, De la musique en Sociologie, Paris, éd. EAP, coll. «Psychologie et Pédagogie de la musique », 1993 ; Emmanuel GRYNSZPAN, Bruyante techno, Nantes, Mélanie Setun, coll. " Musique et Société », 1999 ; Emmanuel GRYNSZPAN, Une fête parallèle, p. 89-103, in Revue Chimères $n^{\circ} 40$, Paris Automne 2000 ; Antoine HENNION, La Passion musicale, une sociologie de la médiation, Paris, Metaillé, 1993 ; T. COLOMBIE, N. LALAM, M. SCHIRAY, Drogues et techno, les trafiquants de rave, Paris, Stock, 2000 ; Jean-Yves LELOUP, Jean-Philippe RENOULT, Pierre Emmanuel hastoIN, Ariel KYRou, Global Techno, Poitiers, Éd. du Camion Blanc, 1999 ; Éric MOLIÈRE, La Musique techno comme fête musicale, p. 217-258 in A.M. Green L'harmattan, Paris 1997 ; Sandy QUEUDRUS, Un maquis techno. Modes d'engagements et pratiques sociales dans la free-party, Paris, Irma, 2000 ; Étienne RACINE, Le Phénomène techno, clubs, raves, freeparty, Paris, Images, 2002 ; D. RICHARD, J.-L. SENON, Dictionnaires des drogues, des toxicomanies et des dépendances, Paris, Bordas, 1999 ; Richard SCHUSTERMANN, L'Art à l'état vif. La pensée pragmatiste et l'esthétique populaire, Paris, Les Éditions de la Nuit, 1992. Revues :

Art Press, « Spécial Techno : anatomie des cultures électroniques », H.S. n 19, 1998 ; Télérama, H.S., « La folie des années Pop », éd. France. F. Ter, 1999 ; Le Mouvement Pop, Lausanne, Bibliothèque Laffont, GT des grands thèmes, 1975 ; Revue Sociétés, «Effervescence Techno », sous la dir. de S. HAMPARTZOUMIAN, n 65, De Boeck/Université, 1999/3 ; La Musique techno : 1 et 2 actes du colloque du 22-23 Janvier 1998, Poitiers, Le confort moderne ; Cultures en mouvement, Hip-Hop, techno, Rythmes de Passage, Octobre $1999, n^{\circ} 21$.

\section{RÉSUMÉS}

Les rassemblements techno occupent beaucoup de nos pensées, de notre imaginaire, de nos discours. Que penser de cette masse de jeunes qui succombent des heures durant aux charmes des musiques électroniques?

Que penser de ces substances, quelles soient chimiques ou naturelles, qui mutent les corps, les consciences et qui deviennent l'espace d'une nuit les compagnons inséparables?

C'est là un fait troublant et intrigant car, dans ces poches émotionnelles que sont les Rave Party, tout se mélange, aussi bien la passion pour la musique que la passion des drogues. Tout se mélange car aussi tout se détourne, se recycle, y compris ces moments d'étouffements où se consument insidieusement tous ces désirs ardents.

Dans ce contexte en mouvement, que veut dire encore fêter, s'amuser, danser, se divertir et tout simplement être ? Est-ce tout simplement d'un Être en transition, d'un être en devenir dont il est question?

Techno parties occupy much of our minds, imaginaries and speeches. What to think of this heap of youngsters succumbing several hours to electronics music's charm? What to think about drugs, naturals or designers drugs, that transform bodies, consciences, and are becoming during nights inseparable playmate? It is a trouble story, a schemer act, because in this emotional 
parties, all are mixed, also music passion and drugs excitation. All are mixed because all are diverted, recycled, included this suffocated moments where all is burning all of passionate desire. In this context, what means this repression, this condemnation? In the right what mean celebrating, having fun, dancing, or simply being?

But here perhaps we talk about hybrid human, that is torn between perfect fair, and virtual fair. But this transitional being can become also from a driving back of the ubris. Our thesis wants to answer to these questions. 